\title{
A SYSTEM AND A DEVICE FOR ISOLATING CIRCULATING TUMOR CELLS FROM THE PERIPHERAL BLOOD IN VIVO
}

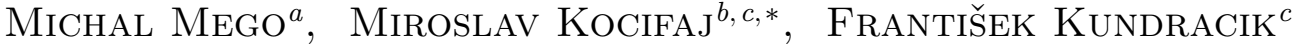 \\ a Department of Oncology, Comenius University, Medical Faculty and National Cancer Institute, Klenová 11, \\ 83310 Bratislava, Slovak Republic \\ ${ }^{b}$ ICA, Slovak Academy of Sciences, Dúbravská Cesta 9, 84503 Bratislava, Slovak Republic \\ ${ }^{c}$ Faculty of Mathematics, Physics, and Informatics, Comenius University, Mlynská dolina, 84248 Bratislava, \\ Slovak Republic \\ * corresponding author: kocifaj@savba.sk
}

\begin{abstract}
Circulating tumor cells (CTC) play a crucial role in disseminating tumors and in the metastatic cascade. CTCs are found only in small numbers, and the limited amount of isolated CTCs makes it impossible to characterize them closely. This paper presents a proposal for a new system for isolating CTCs from the peripheral blood in vivo. The system enables CTCs to be isolated from the whole blood volume for further research and applications. The proposed system consists of magnetic nanoparticles covered by monoclonal antibodies against a common epithelial antigen, large supermagnets, which are used to control the position of the nanoparticles within the human body, and a special wire made of a magnetic core wrapped in a non-magnetic shell. The system could be used not only for isolating CTCs, but also for in vivo isolation of other rare cells from the peripheral blood, including hematopoietic and/or mesenchymal stem cells, with applications in regenerative medicine and/or in stem cell transplantation.
\end{abstract}

KEYWORDS: circulating tumor cells, in vivo isolation, magnetic nanoparticles.

\section{INTRODUCTION}

Circulating tumor cells (CTCs) play a crucial role in tumor dissemination and in the metastatic cascade. CTCs are very rarely-occurring cells, surrounded by billions of hematopoietic cells in the bloodstream. Recent advances in detection methods have enabled them to be identified reproducibly and further characterized 1]. CTCs consistently showed a prognostic value for several types of cancer, including breast, prostate and colon cancer [2 4]. However, CTCs represent a heterogeneous population of cells with various phenotypes and biological values [1]. Different methods detect different CTC subpopulations with different clinical and biological values. All data on CTCs should therefore be interpreted within the context of the detection method that is used [1]. Despite recent technological advances, we are still only beginning to understand CTC-related processes in tumor dissemination and progression.

Various strategies are used for detecting and characterizing CTCs, including morphological and physical characteristics, such as size and weight, and detecting the expression of specific markers. In carcinomas, CTCs are usually identified on the basis of the expression of epithelial-lineage markers, such as EpCaMs (epithelial cell adhesion molecules) or cytokeratins (cytoskeletal proteins present in epithelial cells) and the absence of a common leukocyte marker (CD45) and/or by the presence of putative tumor-specific antigens (for example MUC1 and HER2) [1.
Due to the small numbers of CTCs, almost all detection methods include enrichment steps (negative selection, including depletion of hematopoietic cells, or positive selection, typically using an anti EpCAM antibody) to increase the detection success rate. Despite this enrichment, there is only a very limited amount of isolated CTCs, and CTCs cannot be characterized more closely. Almost all current detection methods detect CTCs from a limited amount of peripheral blood that is drawn from the venous system, while all CTC isolation steps are performed in vitro. In this paper, we propose a new system and device for isolating CTCs from the peripheral blood in vivo. The system enables more cells to be isolated for further research and applications. A major advantage of the proposed system is that it could isolate CTCs (or other cells of interest) from the whole volume of the blood, whereas existing detection methods isolate CTC from a limited volume of blood.

\section{MATERIAL AND METHODS}

\subsection{Magnetic nanoparticles}

The system with magnetic nanoparticles covered by monoclonal antibodies against a common epithelial antigen (EpCAM) is used to isolate circulating tumor cells from the peripheral blood. Magnetic nanoparticles are used because they can be manipulated easily (held and transported) by means of a magnetic field. Magnetic particles can thus be fixed in a vein for a long time, using permanent supermagnets attached to 


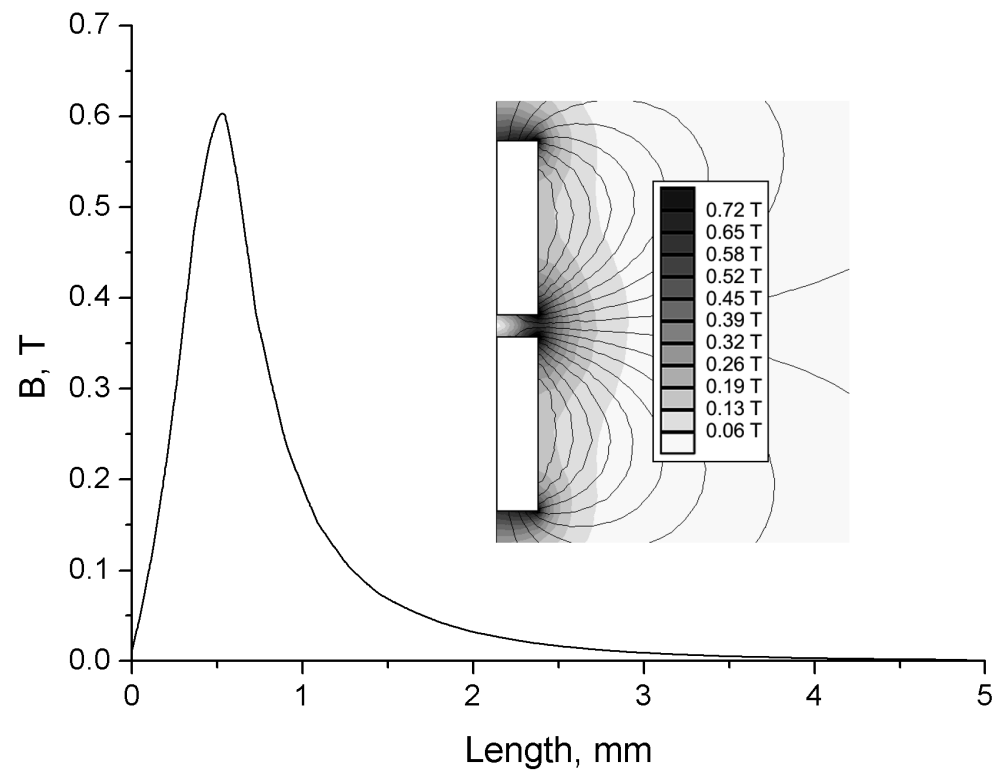

Figure 2. Magnetic induction, $B$, determined along the line perpendicular the wire and crossing the wire between the pellets. Inset: Magnetic field lines in the vicinity of the wire.

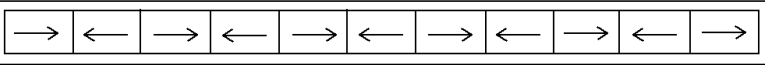

Figure 1. Special wire containing supermagnetic pellets in a non-magnetic mantle.

the arm. This approach is likely to be more comfortable for patients. The magnetic force, $F_{\text {mag }}$, acting on the nanoparticles should be strong enough in relation to the viscous force, $F_{\text {vis }}$, caused by the blood flow. Nacev et al. [5] have analyzed the diffusion effects and the velocity profiles of venous blood, and have shown that the ratio $F_{\text {mag }} / F_{\text {vis }}>5 \cdot 10^{-5}$ for typical viscous forces in the central parts of the vein. Because there is continuous lowering of the blood velocity from the central parts of a vein to its walls, a fixed nanoparticle layer will be formed at the edges - i.e., at the venous walls. The magnetic force acting on a nanoparticle is proportional to its volume, so large-sized nanoparticles are usually preferred for this application. In cases that are important for practically applications, commercially available particles about $30 \mathrm{~nm}$ in diameters would be a suitable choice.

\subsection{MAGNETS}

An external magnetic field is an advantageous concept, since it prevents magnetically guidable nanoparticles spreading over the blood vessels. Large supermagnets are used to control the position of the nanoparticles, and to prevent them moving beyond the area of the arm. The nanoparticles are exposed to circulating tumor cells, and CTCs are picked up by binding them to an EpCAM antibody on the surface of each nanopar- ticle. By manipulating them with large magnets, the nanoparticles can easily be transported towards the venous cannula after the in vivo incubation period.

\subsection{A Wire With A MAGNetic CORE}

A special wire made of a magnetic core wrapped in a non-magnetic shell (Figure 1) is inserted into the cannula, and the large magnets are removed. The nanoparticles with CTCs are attracted by the wire, and are then transferred from the cannula to a tube with an appropriate medium, where the particles scatter within a short time. Before further analysis, the magnetic core should be removed. To make this procedure possible, the wire has to produce a magnetic force large enough to act on the nanoparticles, but low enough to interact with the large magnets. Since the magnetic force is proportional to the magnetic field gradient, the magnetic core can be advantageously made as a chain of supermagnetic pellets with opposite orientations. In this type of configuration the magnetic field of the pellets is pulled out of the wire and large gradients are produced near the touching pellets. Since the force-vector between the pellets and the large magnets changes its direction, the resulting net force between the whole wire and the large magnets is relatively small. If the individual pellets are well separated (e.g., equispaced), rather than glued together, the wire becomes flexible enough for use in in vivo manipulation.

We used a software implementation of the finiteelement method [6] to analyze the wire shown in Figure 1. Pellets $1 \mathrm{~mm}$ in diameter and $2 \mathrm{~mm}$ in length made from standard neodymium magnets were considered. Figure 2 demonstrates the magnetic induc- 


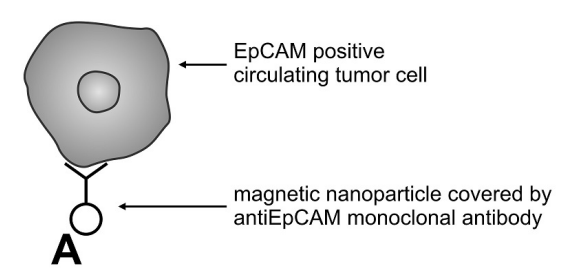

(a)

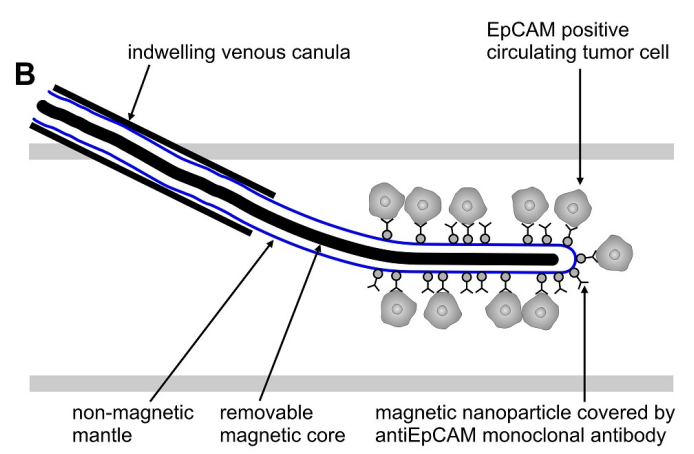

(c)

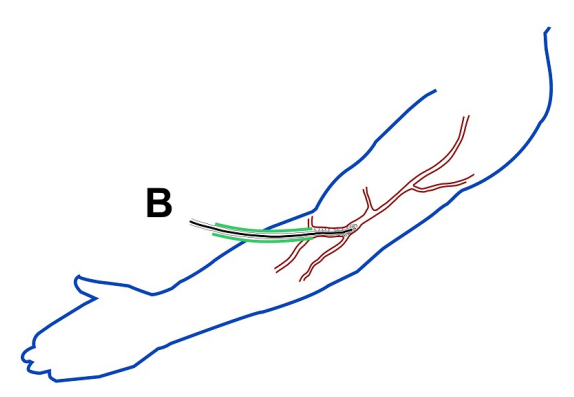

(e)

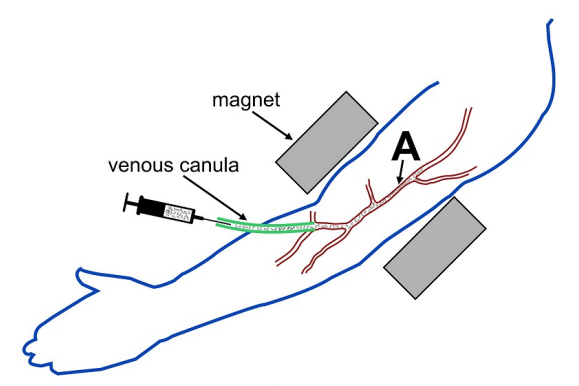

(b)

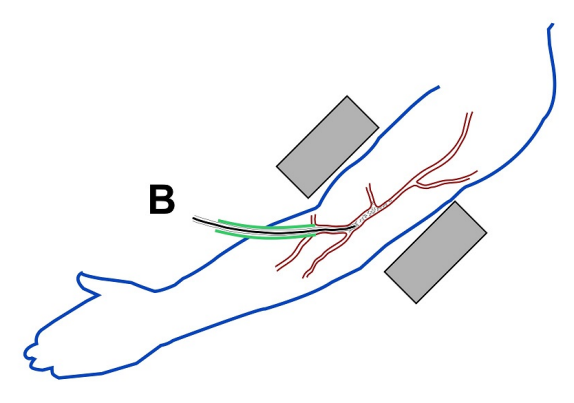

(d)

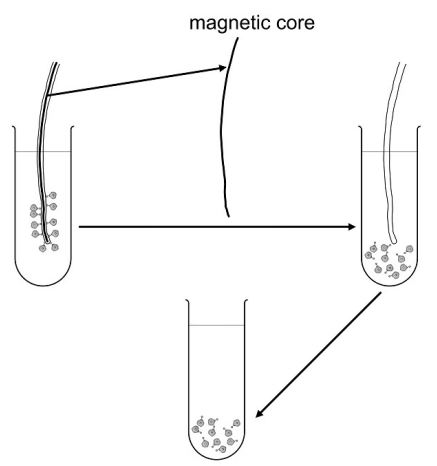

(f)

Figure 3. Proof of concept.

tion, $B$, computed along a line perpendicular to the wire and crossing the wire between the pellets (the length is measured from the axis of symmetry). A magnetic field gradient of $500 \mathrm{~T} / \mathrm{m}$ was found at a distance of $1 \mathrm{~mm}$ from the center of the wire $(0.5 \mathrm{~mm}$ above the surface of the pellets). Magnetic induction exceeding $0.2 \mathrm{~T}$ is large enough to saturate the magnetization of the nanoparticles in this region [7].

The magnetic properties of $\mathrm{Fe}_{3} \mathrm{O}_{4}$ nanoparticles $30 \mathrm{~nm}$ in size [7] were taken into consideration when determining the magnetic force: density of $5 \mathrm{~g} / \mathrm{cm}^{3}$ and magnetic saturation of $20 \mathrm{emu} / \mathrm{g}=100 \mathrm{emu} / \mathrm{cm}^{3}=$ $10^{5} \mathrm{~A} / \mathrm{m}$. The magnetic force acting on a nanoparticle with radius $r$ is

$$
F_{\mathrm{mag}}=M V \operatorname{grad} B=\frac{4 \pi M r^{3} \operatorname{grad} B}{3},
$$

where $M$ is the magnetization of the particle and $V$ is the volume of the particle. Using the parameters mentioned above, we obtain $F_{\text {mag }}=5.7 \cdot 10^{-15} \mathrm{~N}$. The viscous force in a non-turbulent flow can be expressed as follows:

$$
F_{\text {vis }}=6 \pi r \eta v
$$

where $\eta$ is the dynamic viscosity of the blood plasma and $v$ is the velocity of the blood at the center of the vein. Using typical values [8] $\eta=1.3 \cdot 10^{-3} \mathrm{Pas}$ and $v=10 \mathrm{~cm} / \mathrm{s}$, we obtained $F_{\text {vis }}=3.7 \cdot 10^{-11} N$. A ratio of $F_{\text {mag }} / F_{\text {vis }}=1.5 \cdot 10^{-4}$ is sufficient to hold the nanoparticles near the wall of the magnetic wire.

\section{Results}

This is a new system and device for isolating circulating tumor cells from the peripheral blood in vivo. The system is based on isolating CTCs in vivo, using magnetic nanoparticles covered by a monoclonal antibody (Figure 3A.) Magnetic nanoparticles (A) covered by monoclonal antibodies against a common epithelial antigen are slowly injected into the cubital vein (or into another appropriate vein) through the 
venous cannula. The position of these nanoparticles is controlled by large magnets, and their movement is limited to the area of the arm (or to another area of interest) (Figure 3B). Nanoparticles are exposed to the circulating tumor cells, and CTCs are picked up by binding them to an EpCAM antibody on the surface of a nanoparticle. The special wire (B) consists of a magnetic core covered by a non-magnetic mantle. The wire is used for removing nanoparticles with the CTCs attached to them from the bloodstream (Figure $3 \mathrm{C}$ ). After in vivo incubation, the nanoparticles are moved closer to the venous cannula by moving the large magnets. Then a special wire (B) is inserted through the cannula (Figure 3D). Subsequently, the large magnets are removed and the nanoparticles are attracted by a magnetic wire. Then the magnetic wire covered with nanoparticles with CTCs attached to them is removed from the cannula (Figure $3 \mathrm{E}$ ). The wire is placed into the tube with an appropriate medium. The magnetic core is removed, and the nanoparticles with CTCs attached to them are released into the medium for further analysis (Figure $3 \mathrm{~F}$ ).

\section{Discussion}

Numerous methods are used for isolating CTCs from the peripheral blood, with various levels of success, in detecting CTCs, ranging from 1 cell $/ 7.5 \mathrm{~mL}$ of blood to several thousand, but with most of the methods the number of CTCs that are detected is very small (1, $9,10)$. Almost all of these methods isolate CTCs from a limited volume $(5-30 \mathrm{~mL})$ of peripheral blood. CTCs occur in small numbers in the blood, and a limited number of CTCs could be used for further biological characterization. Our system and device overcome this limitation by utilizing in vivo isolation of CTCs. In comparison with a similar system [11, the main advantage of our system is that the nanoparticles float in the bloodstream, and are therefore exposed to CTCs from the whole volume of the blood. These nanoparticles are exposed to the blood not just at the site where the venous cannula is inserted (as is the case in [11]), but they can be moved to large veins with a bigger blood flow, so they can be exposed to more CTCs from the whole blood. Moreover, the nanoparticles can be exposed to the blood flow for several hours, so they can be washed with the whole volume of the blood several times before they are extracted from the bloodstream. Their positions are controlled by external magnets, and the nanoparticles can be placed in any vein of the human body. Another advantage is that the blood does not leave the blood vasculature, so our method is not associated with any blood losses, even when there is repeated isolation of CTCs. Unlike apheresis methods for CTC isolation, the system that we propose here does not need blood anti-coagulation, and the blood is not discharged outside the blood vasculature into any artificial equipment. The method is therefore safer and more comfortable for patients. In addition, the patient is not bed bound. The system can be moved while the patient is exercising, and normal activities can be performed during the procedure.

One of the limitations of the proposed method is that it utilizes positive selection using an anti-EpCAM antibody. CTCs without EpCAM expression, including those undergoing the epithelial-to-mesenchymal transition, are therefore not detected by this method. However, depending on the antibody that is utilized on the surface of the nanoparticles, this system could be used for detecting other rare cells present in the peripheral blood, including hematopoietic stem cells.

This system has several potential applications. For example, it can be used in CTC research to better characterize CTCs, to identify therapeutic targets on CTCs, to perform molecular profiling of tumors that are seeded by CTCs, to study the mechanism of drug resistance, and/or to develop cancer vaccines from the predominant CTC clones. Other potential applications include in vivo isolation of dendritic cells for developing tumor vaccines, or mesenchymal stem cells with applications in regenerative medicine and/or in stem cell transplantation. In addition, the system can be utilized for detecting and isolating any cell of interest present in the blood and/or in another body fluid, with potential uses in translational research, personalized medicine and treatment.

In conclusion, our new system for isolating CTCs from the peripheral blood in vivo enables CTCs to be isolated from the whole volume of the blood for further research and applications. In addition to its use for isolating CTCs, the system could be used for isolating other rare cells from the peripheral blood in vivo, including hematopoietic and/or mesenchymal stem cells, with applications in regenerative medicine and/or in stem cell transplantation.

\section{ACKNOWLEDGEMENTS}

The methods described in this paper present a patent application submitted by Miroslav Kocifaj and Michal Mego. This publication is an outcome of project 1/0724/11, funded by the Slovak Grant Agency VEGA, and project APVV-0016-11, supported by the Slovak Research and Development Agency. This work also received support from the Slovak National Grant Agency VEGA (grant $2 / 0002 / 12$ ). The work presented here has also been supported by Research \& Development Operational Programme project 26240120026 , funded by the ERDF.

There are no competing interests.

The study involves no human subjects.

\section{REFERENCES}

[1] Mego M, Mani SA, Cristofanilli M, Molecular mechanisms of metastasis in breast cancer - clinical applications. Nat. Rev. Clin. Oncol. 7, 693-701 (2010). DOI:10.1038/nrclinonc.2010.171

[2] Cristofanilli M, Budd GT, Ellis MJ, Stopeck A, Matera J, Miller MC, Reuben JM, Doyle GV, Allard WJ, Terstappen LW, Hayes DF, Circulating tumor cells, disease progression, and survival in metastatic breast cancer. N. Engl. J. Med. 351, 781-791 (2004). DOI:10.1056/NEJMoa040766 
[3] Cohen SJ, Punt CJ, Iannotti N, Saidman BH, Sabbath KD, Gabrail NY, Picus J, Morse M, Mitchell E, Miller MC, Doyle GV, Tissing H, Terstappen LW, Meropol NJ, Relationship of circulating tumor cells to tumor response, progression-free survival, and overall survival in patients with metastatic colorectal cancer. J. Clin. Oncol. 26, 3213-3221 (2008). DOI:10.1200/JCO.2007.15.8923

[4] de Bono JS, Scher HI, Montgomery RB, Parker C, Miller MC, Tissing H, Doyle GV, Terstappen LW, Pienta KJ, Raghavan D, Circulating tumor cells predict survival benefit from treatment in metastatic castrationresistant prostate cancer. Clin. Cancer Res. 14, 6302-6309 (2008). DOI:10.1158/1078-0432.CCR-08-0872

[5] Nacev A, Beni C, Bruno O, Shapiro B, Magnetic nanoparticle transport within flowing blood and into surrounding tissue. Nanomedicine 5, 1459-1466 (2010). DOI:10.2217/nnm.10.104

[6] Meeker D. Finite elements method magnetics version 4.2 user's manual. On line:

http://www.femm.info/Archives/doc/manual42.pdf (2013-12-20)

[7] Parvin K, Ma J, Ly J, Sun XC, Nikles DE, Sun K, Wang LM, Synthesis and magnetic properties of monodisperse Fe3O4 nanoparticles. J. Appl. Phys. 95, 7121-7123 (2004). DOI:10.1063/1.1682783
[8] Klabunde RE. Cardiovascular Physiology Concepts. Published by Lippincott Williams \& Wilkins ISBN 9781451113846 (2011).

[9] Allard WJ, Matera J, Miller MC, Repollet M, Connelly MC, Rao C, Tibbe AG, Uhr JW, Terstappen LW.

Tumor cells circulate in the peripheral blood of all major carcinomas but not in healthy subjects or patients with nonmalignant diseases. Clin Cancer Res 10:6897-6890 (2004). DOI:10.1158/1078-0432.CCR-04-0378

[10] Pachmann K, Camara O, Kavallaris A, Krauspe S, Malarski N, Gajda M, Kroll T, Jörke C, Hammer U, Altendorf-Hofmann A, Rabenstein C, Pachmann U, Runnebaum I, Höffken K. Monitoring the response of circulating epithelial tumor cells to adjuvant chemotherapy in breast cancer allows detection of patients at risk of early relapse. J. Clin. Oncol. 26, 1208-1215 (2008). DOI:10.1200/JCO.2007.13.6523

[11] Saucedo-Zeni N, Mewes S, Niestroj R, Gasiorowski L, Murawa D, Nowaczyk P, Tomasi T, Weber E, Dworacki G, Morgenthaler NG, Jansen H, Propping C, Sterzynska K, Dyszkiewicz W, Zabel M, Kiechle M, Reuning U, Schmitt M, Lücke K. A novel method for the in vivo isolation of circulating tumor cells from peripheral blood of cancer patients using a functionalized and structured medical wire. Int. J. Oncol. 41, 1241-1250 (2012). 